\title{
PCAF-mediated acetylation of Lin28B increases let-7 biogenesis in lung adenocarcinoma H1299 cells
}

Ting-ting $\mathrm{Qu}^{\dagger}$, Fei Chen ${ }^{\dagger}$, Jing Wang, Yan-jun Zhang, Mo-bin Cheng, Wen-zheng Sun, Yu-fei Shen and Ye Zhang ${ }^{*}$

\begin{abstract}
Background: Lin28B and its paralog Lin28A are small RNA binding proteins that have similar inhibitory effects, although they target separate steps in the maturation of let-7 miRNAs in mammalian cells. Because Lin28B participates in the promotion and development of tumors mostly by blocking the let-7 tumor suppressor family members, we sought to explore the associated mechanisms to gain insights into how Lin28B might be decreased in human cancer cells to increase let-7 levels and reverse malignancy.

Results: We demonstrated that the histone acetyltransferase PCAF, via its cold shock domain, directly interacts with and subsequently acetylates Lin28B in lung adenocarcinoma-derived H1299 cells. RT-qPCR assays showed that both let-7a-1 and let-7g were increased in PCAF-transfected H1299 cells. Lin28B is acetylated by ectopic PCAF and translocates from the nucleus to the cytoplasm in $\mathrm{H} 1299$ cells.

Conclusions: The effects of acetylated Lin28B on let-7a-1 and let-7g are similar to that of stable knockdown of Lin28B in $\mathrm{H} 1299$ cells. The new role of PCAF in mediating Lin28B acetylation and the specific release of its target microRNAs in H1299 cells may shed light on the potential application of let-7 in the clinical treatment of lung cancer patients.
\end{abstract}

Keywords: Lin28B, PCAF, let-7, Acetylation

\section{Background}

Lin28 and the microRNA let-7 were first discovered in $C$. elegans as heterochronic genes that regulate developmental timing [1-3]. In eukaryotes including worms and mammals, Lin28 blocks let-7 expression, whereas let-7 negatively regulates Lin 28 expression by binding to the 3'UTR of Lin28 mRNA, thereby establishing a double negative feedback loop. The Lin28/let-7 axis plays a pivotal role in stem cell biology and the development and control of glucose metabolism, as well as in human diseases $[4,5]$. In mammals, there are two Lin28 paralogs: Lin28A and Lin28B. Although it is structurally similar to Lin28A, Lin28B contains a cold shock domain (CSD) and a retroviral-type $\mathrm{CCHC}$ zinc finger (ZF) motif. Lin28B has a coding extended $\mathrm{C}$ terminus that contains a nuclear

\footnotetext{
* Correspondence: yezhang@ibms.pumc.edu.cn

${ }^{\dagger}$ Equal contributors

State Key Laboratory of Medical Molecular Biology, Department of Biochemistry and Molecular Biology, Institute of Basic Medical Sciences, Chinese Academy of Medical Sciences \& School of Basic Medicine, Peking Union Medical College, 5 Dongdan Santiao, Beijing 100005, China
}

localization signal (NLS) in addition to a nucleolus localization signal (NoLS) between the CSD and ZF domains, both of which participate in the subcellular localization of Lin28B in human cells [6-10]. The expression of Lin28A in the cytoplasm blocks let-7 processing by Dicer and uridylation of pre-let-7 by TUTase [11], whereas Lin28B primarily accumulates in the nucleus, where it binds pri-let-7 miRNAs and blocks the activity of the microprocessor complex $[5,8,11]$. However, the subcellular localization of Lin28B is controversial [4].

Lin28B was first cloned and identified as an overexpressed factor in hepatocellular carcinoma cells [6]. Lin28B is currently known to be involved in the promotion and development of tumors, thus indicating that it may be a potential target in human cancer therapy $[7,12-15]$. A high Lin28A or Lin28B and low let-7 expression pattern is found in approximately $15 \%$ of human cancers [16]. The expression of Lin28B in cancer cells can be activated by transcription factors and epigenetic modifiers, such as Myc, NF-kB and Sirt6 [17-20]; however, much of the underlying mechanism remains unclear. 
Acetylation is an important modification pattern that has been widely investigated in recent years. Protein acetylation is known to participate in regulating multiple cellular processes in normal and cancer cells [21-23]. As a bona fide cancer-related protein, Lin28B is subject to polyubiquitination that leads to the enhancement of let7 biogenesis [24, 25]. However, whether the acetylation of Lin28B affects the let-7 biogenesis involved in tumorigenesis is not yet fully understood.

In this study, we found that knockdown of Lin28B in the human lung adenocarcinoma cell line H1299 abrogated the inhibition of let-7 miRNA. The histone acetyltransferase PCAF was found to directly interact with Lin28B via its CSD, and this interaction facilitated Lin28B acetylation by the HAT domain of PCAF. Most importantly, we demonstrated that the PCAF-mediated acetylation of Lin28B might de-repress the processing of let-7a-1 and let-7g, and these findings shed light on the potential application of acetylated Lin28B for future cancer therapy.

\section{Methods}

\section{Cell culture}

HEK293T, HCT116, MCF7, HeLa, HepG2, and H1299 cells were cultured in Dulbecco's modified Eagle's medium (DMEM) supplemented with 10\% fetal bovine serum (FBS, HyClone) at $37{ }^{\circ} \mathrm{C}$ in $5 \% \mathrm{CO}_{2}$ atmosphere. The HEK293T cells, MCF7, and H1299 cells were stored in our Lab. The HeLa (Cat. \#3111C0001CCC000011) and HepG2 (Cat. \#3111C0001CCC000035) cell lines were purchased from Chinese National Infrastructure of Cell Line Resource (Beijing, China). HCT116 cell line was a gift from Dr. Depei Liu (Institute of Basic Medical Sciences, Chinese Academy of Medical Sciences, Cat.\# 3111C0001CCC000158). Before the experiments, the two cell lines were authenticated on cell micrograph compared to the cell lines on ATCC. HEK293T cells showed 90\% transfect efficiency with GFP-tag plasmid. H1299 cells showed the lack of p53 protein expression by western blot assay. Mycoplasma contamination was detected by the EZ-PCR Mycoplasma Test Kit (Cat. \#20-700-20), a PCR-based mycoplasma test kit, in cell cultures before the experiment. The kit includes a unique reaction mix that contains all the ingredients required for PCR: nucleotides, primers, Taq Polymerase and magnesium. After performing agarose gel electrophoresis, positive samples will yield a 270 bp fragment, but HEK293T and H1299 cell lines not.

We established two stably transfected clones of H1299 cells, in which Lin28B was knocked down by cotransduction of the cells with lentivirus encoding each of the shRNA specific for Lin28B, designated shLin28B-1, shLin28B-2, and shLuc was as a negative control. The shLin28B-1, shLin28B-2, or shLuc were cloned into the
pLKO.1 vector. The shRNA sequences were as follows: shLin28B-1: GCAGGCATAATAAGCAAGTTA; shLin28B-2: GCCTTGAGTCAATACGGGTAA; shLuc: CGCTGAGTACTTCGAAATGTC.

\section{Antibodies}

Antibodies against $\beta$-actin (sc-47778), GAPDH (sc166545), Myc (sc-789), Lamin B (sc-6216) and PCAF (sc-13,124) were purchased from Santa Cruz Biotechnology (Santa Cruz, CA). Antibodies to FLAG (F3165) and M2 (F2426) were purchased from Sigma (St. Louis, MO). Antibodies to Lin28B (4196) and acetyl lysine (9441) were purchased from Cell Signaling Technology (Beverly, MA).

\section{Plasmids, constructs, and transfection}

The FLAG-tagged and GFP-tagged Lin28B eukaryotic expression plasmids and GST-tagged Lin28B prokaryotic expression plasmids were constructed by cloning Lin28B into the pcDNA6-FLAG, pEGFP-C1-GFP and pGEX$4 \mathrm{~T}-1$ vectors, respectively, using a PCR product from a cDNA library derived from HEK293T cells. pCX-FLAGPCAF, pGEX-PCAF-HAT and pCX-FLAG-PCAF- $\triangle$ HAT were as previously described [16]. Truncated fragments of Lin28B were cloned using the PCR product of fulllength GFP-tagged Lin28B. FLAG-Lin28B- $\triangle$ CSD was constructed by deleting the CSD. Myc-tagged PCAF was cloned from FLAG-tagged PCAF into the pCMV-3tag7Myc vector. The eukaryotic expression plasmid of PCAF containing the Cys574Ser mutation was generated by site-directed mutagenesis of Myc-PCAF. The cells were transfected with Vigofect reagent (Vigorous Biotech, Beijing, China) according to the manufacturer's instructions. Assays were performed 3 times each in triplicate, and all results are shown as the mean \pm SD.

\section{Immunofluorescence}

Cells were cultured on glass cover slips and fixed in $4 \%$ paraformaldehyde at room temperature for $10 \mathrm{~min}$, washed three times with PBS for 15 min, permeabilized with $0.25 \%$ Triton $\mathrm{X}-100$ in PBS for $15 \mathrm{~min}$ at room temperature, washed three times as described above and blocked with $1 \%$ BSA blocking solution at $37{ }^{\circ} \mathrm{C}$ for $1 \mathrm{~h}$. The cells were incubated with primary anti-Lin28B antibody and anti-PCAF antibody at 1:50 dilution, anti-Myc antibody at 1:100 dilution and anti-FLAG antibody at 1:500 dilution at $4{ }^{\circ} \mathrm{C}$ overnight and washed three times with PBS. After washing, the cells were incubated with FITC- or TRITC-conjugated secondary antibodies at a dilution of $1: 200$ at $37{ }^{\circ} \mathrm{C}$ for $1 \mathrm{~h}$ and then washed three times with PBS. The coverslips were stained with DAPI, mounted and examined with a laser scanning confocal microscope. 
Co-immunoprecipitation (co-IP) and immunoblot analyses Transiently transfected HEK293T cells were homogenized in lysis buffer (50 mM HEPES pH 7.5, $150 \mathrm{mM}$ $\mathrm{NaCl}, 2 \mathrm{mM}$ EDTA, $2 \mathrm{mM}$ EGTA, 1\% Triton X-100, $50 \mathrm{mM}$ NaF, $5 \mathrm{mM}$ Sodiun Pyrophosphate, $50 \mathrm{mM}$ Sodium $\beta$-glycerophosphate, $1 \mathrm{mM} \mathrm{NaVO}, 1 \mathrm{mM}$ DTT, $1 \mathrm{mM}$ PMSF, $10 \mu \mathrm{g} / \mathrm{ml}$ leupeptin and $10 \mu \mathrm{g} / \mathrm{ml}$ aprotinin). Cell lysates were rotated at $4{ }^{\circ} \mathrm{C}$ for $30 \mathrm{~min}$ and centrifuged at $12,000 \mathrm{rpm}$ for $15 \mathrm{~min}$ to remove insoluble material and incubated with anti-GFP beads or anti-FLAG M2 beads at $4{ }^{\circ} \mathrm{C}$ overnight. The collected beads were then washed three times and boiled in SDS gel-loading buffer for western blot analysis.

\section{GST pull-down assay}

The GST-tagged Lin28B fusion protein was expressed in Escherichia coli and purified using anti-GST beads. GST or GST-tagged Lin28B beads were individually incubated with cell lysates at $4{ }^{\circ} \mathrm{C}$ overnight. Finally, the beads were washed three times, and the bound proteins were analyzed by western blotting.

\section{Quantitative real-time RT-PCR assays (RT-qPCR)}

RT-qPCR assays were carried out as described previously [26, 27]. Specific primers for Lin28B (forward: AGCCCCTTGGATATTCCAGTC and reverse: AATGTGAATTCCACTGGTTCTCCT). The relative expression of let-7a-1 and let-7g was normalized to that of U6 snRNA using the comparative CT method according to the manufacturer's instructions (Bio-Rad CFX Connect Real-Time System). The primer sequences used for let-7 are listed below (F: forward; R: reverse; RT: reverse transcription). Mature let-7a-1 (RT: GTCGTATCCAGTG CAGGGTCCGAGGTATTCGCACTGGATACGACAAC TAT, F: GCCGCTGAGGTAGTAGGTTGTA and R: GT GCAGGGTCCGAGGT), mature let-7g (RT: GTCGTAT CCAGTGCAGGGTCCGAGGTATTCGCACTGGATAC GACTTGACA, F: GCCGCTGAGGTAGTAGTTTGT and R: GTGCAGGGTCCGAGGT), mature mir-16-1 (RT: GTCGTATCCAGTGCAGGGTCCGAGGTATTCG CACTGGATACGACCGCCAA.

F: GCCGCTAGCAGCACGTAAATAT and R: GTGCA GGGTCCGAGGT) and U6 (RT: AAAATATGGAACGC TTCACGAATTTG, F: CTCGCTTCGGCAGCACA and R AACGCTTCACGAATTTGCGT) were used. The experiments were repeated at least three times with statistical analysis for individual experimental groups. All values were expressed as the mean \pm SD.

\section{In vitro acetyltransferase assay}

In vitro acetyltransferase assays were performed as previously described and detected by using western blotting with anti-acetyl lysine antibody [26]. Briefly, recombinant GST-Lin28B and GST-PCAF-HAT2 proteins were expressed in E. coli BL21 and purified using glutathione beads (Amersham Biosciences). For in vitro acetyltransferase assays, $1 \mu \mathrm{g}$ of GST-PCAF-HAT2 and $5 \mu \mathrm{g}$ of GST or GST-Lin28B were incubated in $25 \mu \mathrm{l}$ of acetyltransferase assay buffer $(50 \mathrm{mM}$ Tris, $\mathrm{pH} \mathrm{8,10 \%} \mathrm{gly-}$ cerol, $10 \mathrm{mM}$ butyric acid, $0.1 \mathrm{mM}$ EDTA, $1 \mathrm{mM}$ DTT, and $1 \mathrm{mM}$ PMSF) with $20 \mu \mathrm{M}$ acetyl $\mathrm{CoA}$ at $30{ }^{\circ} \mathrm{C}$ for $1 \mathrm{~h}$. The reaction products were separated via 15\% SDSPAGE and analyzed by western blotting with anti-acetyl lysine antibody.

\section{Preparation of cell fractions}

The cell fractions were prepared as previously described [28], and the prepared cell fractions were separated with $10 \%$ SDS-PAGE and detected by western blotting.

\section{Statistical analysis}

Statistical analysis was performed using two-tailed Student's t-test. All data were shown as mean with standard deviations (SD). Probabilities of $P>0.05$ were considered as no significant (\#), $P \leqq 0.05$ as significant (*) and $P \leqq 0.01$ as highly significant $\left({ }^{* * *}\right)$.

\section{Results}

The effect of Lin28B knockdown on let-7 expression in H1299 cells

Consistent with the literature [8], Lin28B is mainly found to be distributed in the nucleus (Fig. 1a), where it abrogates the expression of let-7 miRNA. To explore the functions of Lin28B in cancer cells, we first established two stably transfected clones of H1299 cells, in which Lin28B was knocked down by co-transduction of the cells with lentivirus encoding each of the shRNA specific for Lin28B, designated sh-Lin28B-1 and sh-Lin28-2. We found that the expression of the Lin28B protein (Fig. 1b) and its mRNA (Fig. 1c) was lower in the Lin28Bknockdown cells (Lin28B-K/D) than in cells mock transduced with shRNA against luciferase (sh-Luc). We then determined the expression of let-7 in H1299 cells and their Lin28B-K/D counterparts and found that knockdown of Lin28B enhanced the biogenesis of let-7 family members detected (let-7a-1, b, c, d, e, f, and g). The results of two representatives of let- 7 miRNAs were shown in Fig. 1d, the let-7a-1 was increased and let-7g was drastically induced in Lin28B-K/D cells relative to their basal levels in the wild-type H1299 cells but mir-16-1 was not induced at all.

\section{PCAF is associated with Lin28B}

In mammalian cells, PCAF acetylates numerous proteins with multiple roles in the normal growth and function of cells, and it is also associated with the occurrence of cancer [21-23]. Previously we reported that PCAF directly interacted with and acetylated Lin28A, the paralog 
a
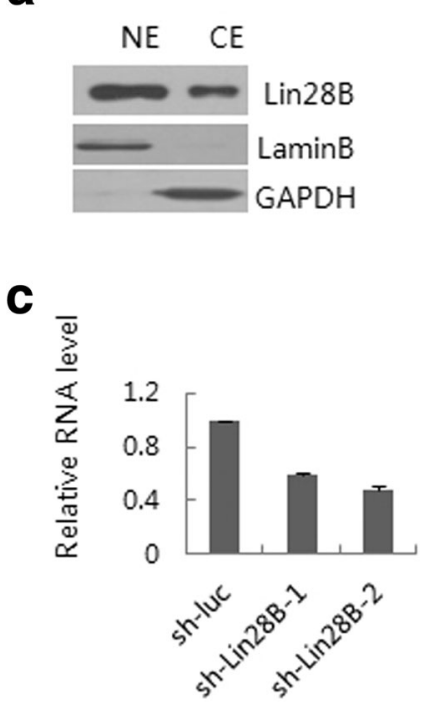

b

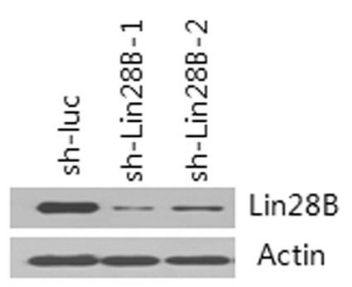

d

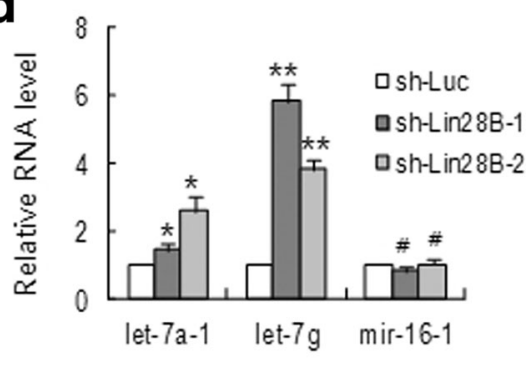

Fig. 1 The effect of Lin28B knockdown on H1299 cells. a The nuclear and cytoplasmic distribution of Lin28B in H1299 cells. Nuclear (NE) and cytoplasmic (CE) extracts were immunoblotted with anti-Lin28B antibody. Lamin B: nuclear marker, GAPDH: cytoplasmic marker. b and c) The expression levels of Lin28B protein (b) and mRNA (c) in H1299 cells with Lin28B stably knocked down. Whole cell extracts (WCEs) from sh-Lin28B-1-, sh-Lin28B-2-, and sh-luc-transduced $\mathrm{H} 1299$ cells were subjected to gel electrophoresis and were blotted with antibodies, as shown on the right. $\beta$-actin: loading control (b); RT-qPCR. U6 snRNA was used as reference for mature miRNAs (c). d RT-qPCR analysis showing the levels of let-7a-1, let-7g and mir-16-1 in the control and Lin28B stable knockdown cells. Sh-luc: sh-RNA specific for luciferase as a negative control. In histograms, each bar represents a mean value \pm S.D. from at least three independent experiments $\left(\#, P>0.05 ;{ }^{*} p \leq 0.05 ;{ }^{*} p \leq 0.01\right)$

of Lin28B [26]. To identify whether PCAF isassociated with Lin28Bwe assessed the interaction between PCAF and Lin28B by co-IP assay. Anti-GFP antibody was applied to co-immunoprecipitate GFP-tagged Lin28B with ectopic FLAG-PCAF in HEK293T cells (Fig. 2a). In addition, in vitro GST-pulldown assays indicated that FLAG-PCAF was pulled-down by GST-Lin28B in the HEK293T cell lysates (Fig. 2b). These results demonstrated an interaction between Lin28B and PCAF. To further identify the binding domain of Lin28B that interacts with PCAF, the coding region of the Lin28B gene was truncated; the representative protein fragments are shown in Fig. 2c. The co-IP assay revealed that the CSD of Lin28 (Fig. 2d, L1 \& L4) was essential in mediating the interaction between Lin28B and PCAF. Similar results from in vitro GST-pulldown assays also indicated that the CSD of Lin28B mediated its binding with PCAF (Fig. 2e). Additionally, endogenous Lin28B- and PCAFexpressing H1299 cells were cultured on glass cover slips and treated with antibodies for immunofluorescence staining. Our results showed that Lin28B and PCAF colocalized in the nucleus of H1299 cells (Fig. 2f).

PCAF acetylates Lin28B and involves in de-pression of let-7 To explore whether Lin28B is acetylated by PCAF, an in vitro acetyltransferase assay was performed, as previously reported [26]. Using anti-acetyl lysine (AcK) antibody, we found that GST-Lin28B was acetylated only in the presence of the purified GST-fused HAT2domain of PCAF (GST-PCAF-HAT), whereas PCAF was auto-acetylated (Fig. 3a). The expression constructs for FLAG-Lin28B were co-transfected into HEK293T cells with either Myc-PCAF or HAT-domain-deleted MycPCAF (Myc-PCAF- $\triangle$ HAT). Ectopically expressed Lin28B was acetylated by Myc-tagged PCAF in the cells, whereas no acetylation of Lin28B was observed with the HATdomain-deleted PCAF (Fig. 3b). To explore the functional role of PCAF compared with its HAT-domain-deleted counterpart in non-modified H1299 cells, RT-qPCR analysis was performed, and elevated expression levels of the let-7a-1 and let-7g miRNAs were found in the presence of PCAF but not the HAT-domain-deleted PCAF, whereas mir-16-1 was not induced by PCAF transfection (Fig. 3c). These results suggested that the acetylation of Lin $28 \mathrm{~B}$ by PCAF is a critical event that abrogates the inhibitory effect of native Lin28B on the biogenesis of let-7a-1 and let-7g miRNAs in H1299 cells.

We have previously reported that the protein stability of Lin28A, the paralog of Lin28B, decreases when it is acetylated by PCAF [26]; however, no apparent change was observed in the protein level of Lin28B in the cells transfected with PCAF (data not shown). Co-IP assays showed that PCAF- $\triangle$ HAT still interacted with Lin28B in cells (Fig. 3d).

To reveal the mechanism underlying the PCAFmediated acetylation of Lin $28 \mathrm{~B}$ and its effect on the 


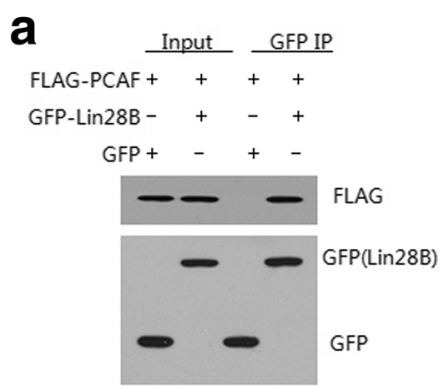

C
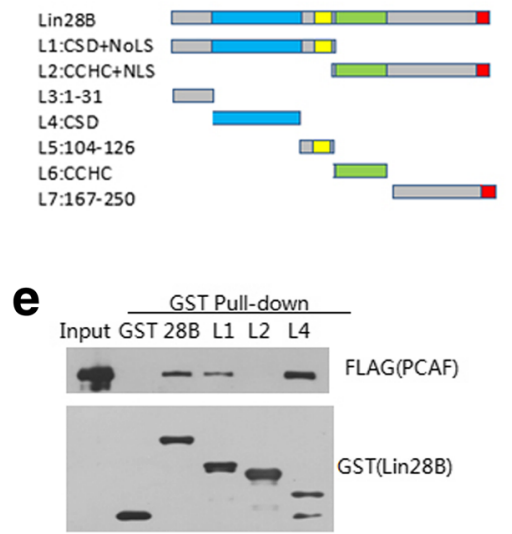

b
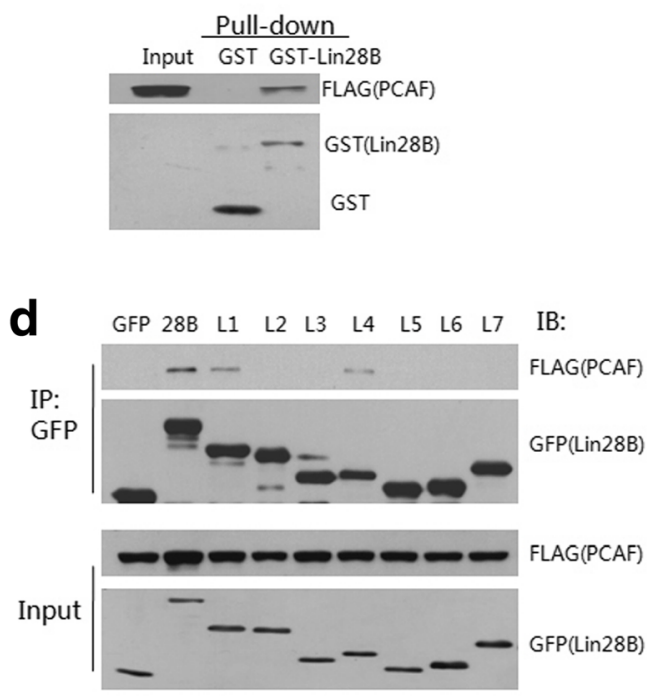

f

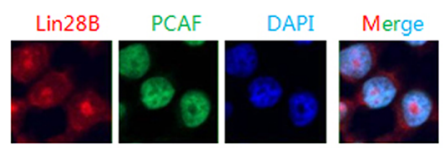

Fig. 2 Lin28B interacts with PCAF via its cold shock domain. a Co-IP of the co-transfected PCAF-FLAG and Lin28B-GFP expression constructs in HEK293T cells. WCEs were IP-ed with anti-GFP antibody, and immunoblotted (IB) with the antibodies shown on the right. WCEs without immunoprecipitation are shown as Input. b GST-pulldown assay. Lin28B-GFP or GFP alone was individually incubated with WCEs of HEK293T cells ectopically expressing FLAG-PCAF, and the samples were subjected to gel electrophoresis and were blotted with antibodies shown on the right. c Schematic representation of the truncation fragments of Lin28B. Digits indicate the positions of the amino acids at the terminus of each fragment. $\mathbf{d}$ Domain-specific binding of Lin28B with PCAF. Each of the expression constructs of Lin28B and its individually truncated fragments was tagged with GFP (labeled as L1 L7) and individually co-transfected with PCAF-FLAG in HEK293T cells. WCEs of each transfected sample was IP-ed with anti-GFP antibody and then subjected to gel electrophoresis and was blotted with antibodies shown on the right. c GST-pull down assay identifying the interactions between specific Lin28B domains and PCAF in vitro. As shown in (d), each of the GST-tagged Lin28B and the related samples indicated at top was incubated with WCEs containing FLAG-tagged PCAF, and samples were then analyzed by western blotting with antibodies against GST and FLAG, as indicated. f Co-localization of Lin28B and PCAF in human cells. Immunofluorescence images showing endogenous Lin28B and PCAF in H1299 cells

biogenesis of let-7, we first determined the expression of Lin28B in different cell types and detected it in three cell lines, H1299, HepG2, and HEK293T, but it was not detectable in other cell lines including HCT116, MCF7, and HeLa cells (Fig. 3e). However, unlike HEK293T cells, Lin28B was mainly distributed in the nucleus of H1299 cells (last group of cells in Fig. 3e). Furthermore, as compared with localization of Lin28A mostly in the cytoplasm but not in the nucleus $[8,26], \operatorname{Lin} 28 \mathrm{~B}$ was mainly located in nucleus in these cells, and acetylation of Lin28B by ectopic PCAF enabled its translocation from the nucleus to the cytoplasm of H1299 cells, as shown by the results of immunofluorescence assays (Fig. 3f) and western blot assays on nuclear/cytoplasmic extracts (Fig. 3g). The distribution of endogenous Lin28B detected by anti-Lin28B is similar with that of ectopic Lin28B staining with anti-FLAG in H1299 cells (Fig. 2f vs Fig. 3f), which is confirmed with results of western blot for the fractions of nuclear/cytoplasmic
(Fig. 1a vs the first two lines of Fig. 3g). To analyze the effects of PCAF activity on Lin28B, we used a Cys574 mutant of PCAF (M-PCAF) and Lin28B- $\triangle$ CSD and detected the cytoplasmic distribution of Lin28B in H1299 cells. The results showed that M-PCAF did not increase the cytoplasmic distribution of Lin28B and that the CSDdeleted Lin28B was mainly distributed in the cytoplasm and was not affected by M-PCAF (Fig. 3h). These findings indicated that the acetylation of Lin $28 \mathrm{~B}$ was induced by PCAF, which removed the inhibition on let-7 biogenesis in H1299 cells, as schematically shown in Fig. 3i.

\section{Discussion}

In this report, we demonstrated that knockdown of Lin28B in H1299 cells elevated the level of let-7a-1/g, although this level of let-7a-1 was lower than that of let$7 \mathrm{~g}$ in Lin28B-knockdown cells. The cell line H1299 was used as a model of lung adenocarcinoma and was derived from a lymph node metastasis of the lung. H1299 


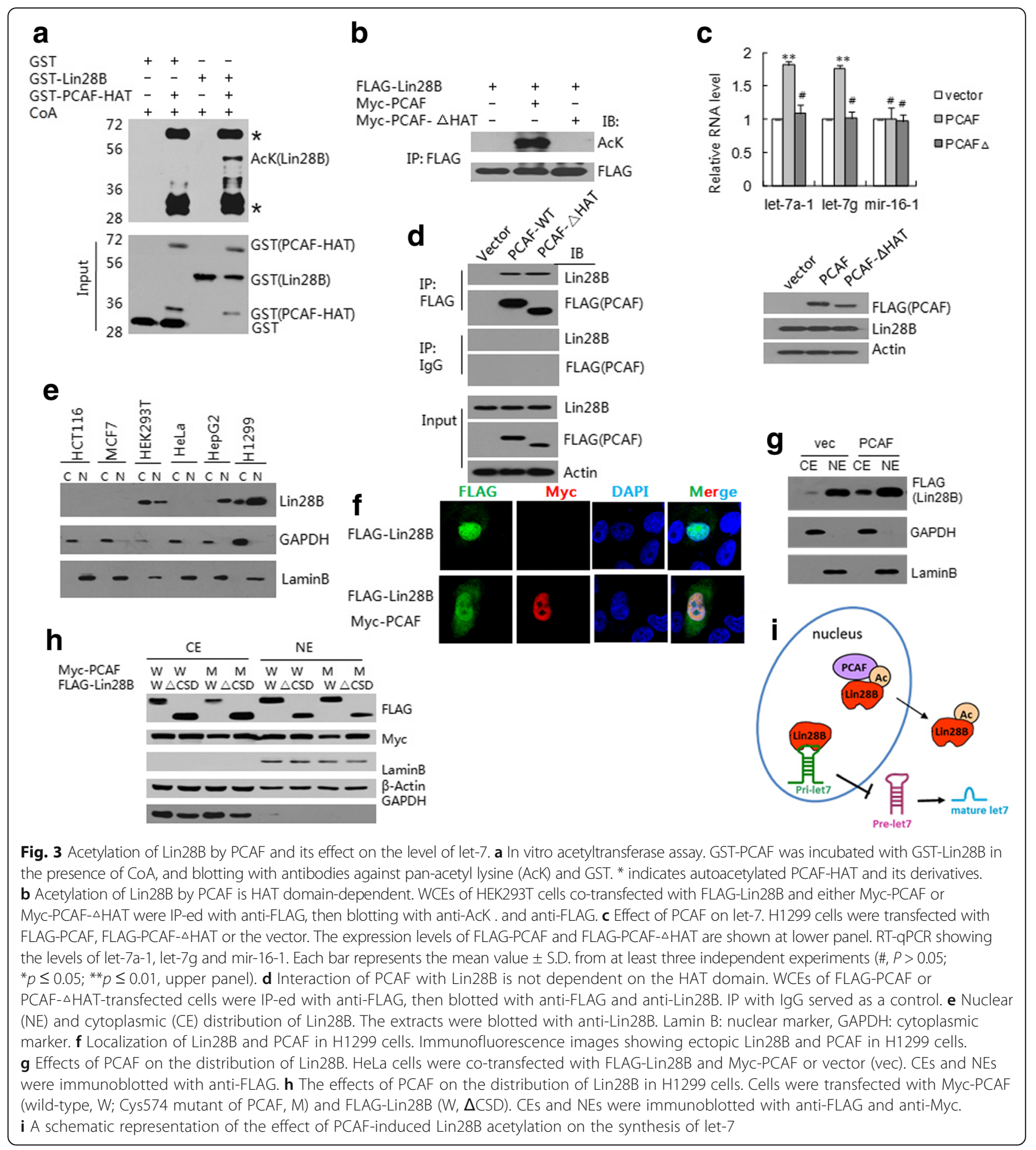

cells express high levels of Lin28B and low levels of let-7 $[8,16]$. Lin28B, after acetylation by PCAF, might translocate from the nucleus to cytoplasm in $\mathrm{H} 1299$ cells and it may be further involved in the de-repression of let- 7 biogenesis. These findings provide new insights into the potential application of acetylated Lin28B in mediating let-7 biogenesis, which may serve as a novel therapeutic approach for lung cancer.
In humans, there are twelve let-7 family members located at eight different chromosomal loci. Lin28A/B selectively represses the expression of let-7 miRNAs [11, 29-31]. It is well known that cytoplasmic Lin28A first binds to the conserved terminal loop of pre-let-7 and then recruits TUT4 for polyuridylation, thereby blocking Dicer cleavage [4, 5]. Although the precise mechanism underlying the Lin28B-mediated inhibition 
of let-7 remains controversial, it has been demonstrated that Lin28B expression and let-7 loss almost invariably correlate with poor prognosis [4]. Activation of Lin28B expression in cancer cells can be triggered by upstream transcriptional factors, such as c-Myc $[18,19]$ and NF$\kappa B$ [20]. In addition, it has been reported that Merlin/ NF2 is a key regulator of Lin28B localization and let-7 biogenesis in response to cell-cell contact [32]. Our data suggested that acetylation of Lin28B by PCAF may affect Lin28B localization and let-7 biogenesis.

Although we have previously reported that the protein level of Lin28A had decreases in PCAF-transfected cells [26], Lin28B was not substantially changed at the protein level (data not shown). Both Lin28A and Lin28B interact with PCAF via their CSD, and let-7 binds to the 3'UTR of both Lin 28 paralogs, thereby repressing Lin 28 translation in a negative feedback loop; however, TRIM71, a specific E3 ubiquitin ligase, negatively regulates Lin28B (but not Lin28A) protein levels by ubiquitinating its Cterminal unique region, which is absent in the Lin28A paralog $[24,25]$. It might be interesting to study whether the acetylation of Lin28B by PCAF affects the polyubiquitination of Lin28B at the C-terminal region and may alter its stability.

\section{Conclusion}

In summary, we demonstrated that Lin28B interacts directly with PCAF via its cold shock domain and is acetylated by PACF. The HAT domain of PCAF is indispensable for PCAF mediated acetylation of Lin28B and de-repression of let-7a-1 and let-7g. Lin28B, after acetylation by PCAF, might translocate from the nucleus to cytoplasm in H1299 cells and it may be further involved in the de-repression of let-7 biogenesis. The discovery of a new role of PCAF in mediating Lin28B acetylation and, particularly, in elevating the level of microRNAs in lung adenocarcinoma-derived H1299 cells may shed new light on the potential application of let-7a-1 and/or let-7g in the clinical treatment of lung cancer.

\section{Abbreviations}

AcK: Acetyl lysine; Co-IP: Co-immunoprecipitation; CSD: Cold shock domain; DMEM: Dulbecco's modified Eagle's medium; Lin28B-K/D: Lin28B-knockdown cell lines; NLS: Nucleus localization signal; NoLS: Nucleolus localization signal; RT-qPCR: Quantitative real-time RT-PCR assays; ZF: CCHC zinc finger motif

\section{Acknowledgements \\ We thank Dr. Q.W. Zhai (INS, SIBS, CAS) for kindly providing the PCAF plasmids.}

\section{Funding}

This work was supported by the National Basic Research Program of China (973 Program) (2013CB531301), the National Natural Science Foundation of China (31371305), and Special Funds from State Key Laboratories (2060204). Funding sources had no role in the study design, analysis, or interpretation of the data, writing the manuscript, or the decision to submit the manuscript for publication.

\section{Availability of data and materials}

The datasets used and/or analyzed during the current study are available from the corresponding author on reasonable request.

\section{Authors' contributions}

TQ conceived, designed, performed, and analyzed experiments and also wrote the manuscript. FC, JW, YaZ, MC, and WS performed and analyzed experiments and revised the manuscript. YS conceived and designed experiments and wrote the manuscript. YeZ conceived, designed, and analyzed experiments and wrote the manuscript. All authors have read and approved the final version of this manuscript.

Ethics approval and consent to participate

Not applicable.

\section{Consent for publication}

Not applicable.

\section{Competing interests}

The authors declare that they have no competing interests.

\section{Publisher's Note}

Springer Nature remains neutral with regard to jurisdictional claims in published maps and institutional affiliations.

Received: 21 June 2017 Accepted: 21 December 2017

Published online: 04 January 2018

\section{References}

1. Moss EG, Lee RC, Ambros V. The cold shock domain protein LIN-28 controls developmental timing in C. Elegans and is regulated by the lin-4 RNA. Cell. 1997;88(5):637-46

2. Reinhart BJ, Slack FJ, Basson M, Pasquinelli AE, Bettinger JC, Rougvie AE, Horvitz HR, Ruvkun G. The 21-nucleotide let-7 RNA regulates developmental timing in Caenorhabditis Elegans. Nature. 2000:403(6772):901-6.

3. Slack FJ, Basson M, Liu Z, Ambros V, Horvitz HR, Ruvkun G. The lin-41 RBCC gene acts in the C. Elegans heterochronic pathway between the let-7 regulatory RNA and the LIN-29 transcription factor. Mol Cell. 2000;5(4):659-69.

4. Balzeau J, Menezes MR, Cao S, Hagan JP. The LIN28/let-7 pathway in cancer. Front Genet. 2017;8:31

5. Thornton JE, Gregory RI. How does Lin28 let-7 control development and disease? Trends Cell Biol. 2012;22(9):474-82.

6. Guo Y, Chen Y, Ito H, Watanabe A, Ge X, Kodama T, Aburatani H. Identification and characterization of LIN-28 homolog B (LIN28B) in human hepatocellular carcinoma. Gene. 2006;384:51-61.

7. Molenaar JJ, Domingo-Fernandez R, Ebus ME, Lindner S, Koster J, Drabek K, Mestdagh P, van Sluis P, Valentijn LJ, van Nes J, et al. LIN28B induces neuroblastoma and enhances MYCN levels via let-7 suppression. Nat Genet. 2012;44(11):1199-206

8. Piskounova E, Polytarchou C, Thornton JE, LaPierre RJ, Pothoulakis C, Hagan JP, lliopoulos D, Gregory RI. Lin28A and Lin28B inhibit let-7 microRNA biogenesis by distinct mechanisms. Cell. 2011;147(5):1066-79.

9. Wang YC, Chen YL, Yuan RH, Pan HW, Yang WC, Hsu HC, Jeng YM. Lin-28B expression promotes transformation and invasion in human hepatocellular carcinoma. Carcinogenesis. 2010;31(9):1516-22.

10. Zhou J, Ng SB, Chng WJ. LIN28/LIN28B: an emerging oncogenic driver in cancer stem cells. Int J Biochem Cell Biol. 2013;45(5):973-8.

11. Heo I, Joo C, Cho J, Ha M, Han J, Kim VN. Lin28 mediates the terminal uridylation of let-7 precursor MicroRNA. Mol Cell. 2008;32(2):276-84.

12. Alajez NM, Shi W, Wong D, Lenarduzzi M, Waldron J, Weinreb I, Liu FF. Lin28b promotes head and neck cancer progression via modulation of the insulin-like growth factor survival pathway. Oncotarget. 2012;3(12):1641-52.

13. Hu Q, Peng J, Liu W, He X, Cui L, Chen X, Yang M, Liu H, Liu S, Wang H. Lin28B is a novel prognostic marker in gastric adenocarcinoma. Int J Clin Exp Pathol. 2014;7(8):5083-92.

14. King CE, Cuatrecasas M, Castells A, Sepulveda AR, Lee JS, Rustgi AK. LIN28B promotes colon cancer progression and metastasis. Cancer Res. 2011;71(12): 4260-8.

15. Madison BB, Liu Q, Zhong X, Hahn CM, Lin N, Emmett MJ, Stanger BZ, Lee JS, Rustgi AK. LIN28B promotes growth and tumorigenesis of the intestinal epithelium via Let-7. Genes Dev. 2013;27(20):2233-45. 
16. Viswanathan SR, Powers JT, Einhorn W, Hoshida Y, Ng TL, Toffanin S, O'Sullivan M, Lu J, Phillips LA, Lockhart VL, et al. Lin28 promotes transformation and is associated with advanced human malignancies. Nat Genet. 2009:41(7):843-8.

17. Kugel S, Sebastian C, Fitamant J, Ross KN, Saha SK, Jain E, Gladden A, Arora KS, Kato Y, Rivera MN, et al. SIRT6 suppresses pancreatic cancer through control of Lin28b. Cell. 2016;165(6):1401-15.

18. Chang TC, Zeitels LR, Hwang HW, Chivukula RR, Wentzel EA, Dews M, Jung J, Gao P, Dang CV, Beer MA, et al. Lin-28B transactivation is necessary for Myc-mediated let-7 repression and proliferation. Proc Natl Acad Sci U S A. 2009;106(9):3384-9.

19. Jiang X, Huang H, Li Z, Li Y, Wang X, Gurbuxani S, Chen P, He C, You D, Zhang S, et al. Blockade of miR-150 maturation by MLL-fusion/MYC/LIN-28 is required for MLL-associated leukemia. Cancer Cell. 2012;22(4):524-35.

20. Iliopoulos D, Hirsch HA, Struhl K. An epigenetic switch involving NF-kappaB, Lin28, Let-7 MicroRNA, and IL6 links inflammation to cell transformation. Cell. 2009;139(4):693-706.

21. Fu M, Wang C, Zhang X, Pestell RG. Acetylation of nuclear receptors in cellular growth and apoptosis. Biochem Pharmacol. 2004;68(6):1 199-208.

22. Mateo F, Vidal-Laliena M, Pujol MJ, Bachs O. Acetylation of cyclin a: a new cell cycle regulatory mechanism. Biochem Soc Trans. 2010;38(Pt 1):83-6.

23. Zhuang S. Regulation of STAT signaling by acetylation. Cell Signal. 2013; 25(9):1924-31.

24. Lee SH, Cho S, Kim MS, Choi K, Cho JY, Gwak HS, Kim YJ, Yoo H, Lee SH, Park JB, et al. The ubiquitin ligase human TRIM71 regulates let-7 microRNA biogenesis via modulation of Lin28B protein. Biochim Biophys Acta. 2014; 1839(5):374-86.

25. Yin J, Kim TH, Park N, Shin D, Choi HI, Cho S, Park JB, Kim JH. TRIM71 suppresses tumorigenesis via modulation of Lin28B-let-7-HMGA2 signaling. Oncotarget. 2016;7:79854

26. Wang $L X$, Wang J, Qu TT, Zhang Y, Shen YF. Reversible acetylation of Lin28 mediated by PCAF and SIRT1. Biochim Biophys Acta. 2014;1843(6):1188-95.

27. Chen C, Ridzon DA, Broomer AJ, Zhou Z, Lee DH, Nguyen JT, Barbisin M, XU $\mathrm{NL}$, Mahuvakar VR, Andersen MR, et al. Real-time quantification of microRNAs by stem-loop RT-PCR. Nucleic Acids Res. 2005;33(20):e179,

28. Xie R, Wang Y, Nie W, Huang W, Song W, Wang Z, Guan X. Lin28B expression correlates with aggressive clinicopathological characteristics in breast invasive ductal carcinoma. Cancer Biother Radiopharm. 2014;29(5):215-20.

29. Viswanathan SR, Daley GQ, Gregory RI. Selective blockade of microRNA processing by Lin28. Science. 2008;320(5872):97-100.

30. Rybak A, Fuchs H, Smirnova L, Brandt C, Pohl EE, Nitsch R, Wulczyn FG. A feedback loop comprising lin-28 and let-7 controls pre-let-7 maturation during neural stem-cell commitment. Nat Cell Biol. 2008;10(8):987-93.

31. Newman MA, Thomson JM, Hammond SM. Lin-28 interaction with the Let-7 precursor loop mediates regulated microRNA processing. RNA. 2008;14(8): 1539-49.

32. Hikasa H, Sekido Y, Suzuki A. Merlin/NF2-Lin28B-let-7 is a tumor-suppressive pathway that is cell-density dependent and hippo independent. Cell Rep. 2016;14(12):2950-61.

\section{Submit your next manuscript to BioMed Central and we will help you at every step:}

- We accept pre-submission inquiries

- Our selector tool helps you to find the most relevant journal

- We provide round the clock customer support

- Convenient online submission

- Thorough peer review

- Inclusion in PubMed and all major indexing services

- Maximum visibility for your research

Submit your manuscript at www.biomedcentral.com/submit

) Biomed Central 